\title{
Cache Performance Analysis of VIRTUALIZED ROUTER ON VirTual CONTENT-CENTRIC NETWORKS
}

\author{
Keiichiro Tsukamoto ${ }^{1}$, Kaito Ohsugi ${ }^{1}$, Hiroyuki Ohsaki ${ }^{2}$, \\ Toru Hasegawa ${ }^{1}$ and Masayuki Murata ${ }^{1}$ \\ ${ }^{1}$ Graduate School of Information Science and Technology, \\ Osaka University, Osaka, Japan \\ ${ }^{2}$ Department of Informatics, School of Science and Technology, \\ Kwansei Gakuin University, Hyogo, Japan
}

\begin{abstract}
Content-centric networking (CCN) is one of the major proposals for realizing information-centric networking. CCN routers cache forwarded data in a buffer memory called the ContentStore (CS). Virtual content-centric networking (VCCN), which enables the construction of multiple virtual networks (called VCCN slices) on a content-centric network, has been recently proposed. When multiple VCCN slices are constructed, the performance of each VCCN slice and that of the entire network are strongly affected by the $C C N$ routers' CS allocation to VCCN router instances in VCCN slices. In this paper, we analyze the effects of CS allocation methods and content request patterns in VCCN slices on the performance of each VCCN slice and that of the entire network. Through several numerical examples, we show that when content request patterns are heterogeneous, a hybrid resource allocation method is effective in terms of both network fairness for VCCN slices and overall network performance.
\end{abstract}

\section{KEYWORDS}

CCN (Content-Centric Networking), Router Virtualization, Resource Allocation, Markov Chain, LRU (Least Recently Used)

\section{INTRODUCTION}

Content-centric networking $(\mathrm{CCN})$, in which a router routes a packet based on an identifier assigned to content, is one of the major proposals for realizing information-centric networking [1]. In $\mathrm{CCN}$, a request packet from a user, called an Interest packet, is routed between $\mathrm{CCN}$ routers according to the longest prefix matching the requesting content identifier. If the Interest packet is successfully delivered to the source, the content packet, called a Data packet, is sent back to the user by traversing the path of the Interest packet. CCN routers cache forwarded Data packets in a buffer memory called the ContentStore (CS). When a CCN router receives an Interest packet for a cached Data packet, it returns the cached packet so that the average content delivery time and the amount of traffic over the network can be reduced.

Virtual content-centric networking (VCCN), which enables the construction of multiple virtual networks (called VCCN slices) on a content-centric network, has been recently proposed [2]. 
International Journal of Next-Generation Networks (IJNGN) Vol.5, No.4, December 2013

VCCN slices are constructed by operating a $\mathrm{CCN}$ router as multiple, logically independent VCCN router instances and by logically connecting VCCN router instances that are not adjacent in the network.

When multiple VCCN slices are constructed, the performance of each VCCN slice and that of the entire network are strongly affected by the CCN routers' resource allocation to VCCN router instances in VCCN slices. Several previous studies have shown clearly that, in CCN, the effectiveness of content caching depends strongly on the content request pattern experienced by the CS of a CCN router [3-5]. Hence, the performance of each VCCN slice and that of the entire network depend strongly on how a CCN router allocates its CS to VCCN router instances on VCCN slices that have different content request patterns.

In this paper, we analytically investigate the effect of CS allocation methods and content request patterns in VCCN slices in terms of the network fairness for VCCN slices and overall network performance. We focus on the effects of the content popularity slope and the content request ratio of each VCCN slice, which are the main features of a content request pattern and which significantly affect the effectiveness of content caching in particular.

In this paper, we focus on three types of CS allocation methods: an exclusive method, a shared method and a hybrid method. In the exclusive method, each VCCN router instance within a CCN router monopolizes a given part of its CS. In the shared method, all VCCN router instances within a CCN router use its entire CS jointly. In the hybrid method, several VCCN router instances within a CCN router are assigned their own parts of its CS and other instances jointly use the remaining CS. Previous studies of the effects of content caching on content-centric networks have focused only on the exclusive and shared methods [6-9]. However, when content request patterns are heterogeneous, these two methods can barely maintain a balance between network fairness for VCCN slices and overall network performance. Hence, we conjectured that a hybrid method, which has the characteristics of both the exclusive and shared approaches, might be a useful CS allocation method on a content-centric network in which there are multiple content request patterns in VCCN slices. In this paper, we quantitatively compare a hybrid method with the two existing methods in terms of the fairness for VCCN slices and the overall network performance.

The main contribution of this paper is twofold. First, we develop a mathematical model of virtualized CCN router for cache performance analysis under arbitrary content request patterns, and derive the cache hit rate for each VCCN router instance and the aggregated cache hit rate of the virtualized CCN router. Second, through numerical examples, we quantitatively show that in di- verse scenarios, the hybrid method can provide desirable trade-offs among the network fairness for VCCN slices and overall network performance.

The organization of this paper is as follows. Section 2 contains a summary of related work. In Section 3, we describe CCN router virtualization and CS allocation to VCCN router instances. In Section 4, our model of a virtualized CCN router which accommodates multiple VCCN router instances is described and analytical results are derived. In Section 5, through several numerical examples, we analyze the effects of CS allocation methods and content request patterns in VCCN slices on the network fairness for VCCN slices and the overall network performance. Finally, in Section 6, we give our conclusions and indicate the direction of future work.

\section{RELATED WORK}

The effect of content caching on content-centric networks where multiple applications or services are running has been investigated in [6-9]. Carofiglio et al. [6, 7] have clarified the role of the CS allocation method (an exclusive method) on the cache hit rate of multiple applications running on 
International Journal of Next-Generation Networks (IJNGN) Vol.5, No.4, December 2013

content-centric networks by means of experiments and simulations. Their results show that an exclusive method can guarantee application performance but it may decrease the overall performance of the entire network. Fricker et al. [8] have evaluated the cache hit rate of multiple services running concurrently on a content-centric network using an approximation proposed by Che et al. [10]. Their results show that allowing a service with a rapid content popularity slope to monopolize the CS raises the cache hit ratio rather than increasing the quantity of CS shared between all the services, when the size of the CS is large. Ohsugi et al. [9] investigated by means of a simulation the effect of CS allocation in CCN router virtualization (an exclusive method) in terms of the average content delivery time for an entire content-centric network on which multiple applications are running. Their results show that exclusive CS allocation in $\mathrm{CCN}$ router virtualization increases the average content delivery time by about $20 \%$ in the worst case and improves network fairness for applications.

These studies show that while a shared method is preferable for maximizing the performance of the entire network, an exclusive method is preferable for improving network fairness for applications or services. However, a hybrid method, which will strike a balance between performance and fairness, has not yet been described or quantitatively evaluated.

The effect of a content request pattern in a content-centric network on caching performance has been investigated in [3,4]. Rossini et al. [3, 4] evaluated by means of simulations the dependence of the cache hit rate on several aspects of network design, such as topology, content size, content popularity, the locality of user requests and the number of repositories. Their results show that the Zipf exponent $\alpha$, representing content popularity, can have a dramatic impact on the performance of the entire network. However, in those studies, CCN router virtualization was not taken into consideration, and the relation between CS allocation methods and content request patterns in VCCN slices has not yet been clarified.

\section{CCN ROUTER VIRTUALIZATION AND CS ALLOCATION TO VCCN ROUTER INSTANCES}

With CCN router virtualization, a single $\mathrm{CCN}$ router is operated as multiple, logically independent VCCN router instances (see Fig. 1 [2]). CCN router virtualization can be easily realized by switching three data structures used for packet routing in $\mathrm{CCN}$ : the forwarding information base (FIB), the CS, and the pending interest table (PIT) [2]. A VCCN router instance routes packets using its own data structures (i.e., FIB, CS and PIT) in the same way that a CCN router does.

A VCCN slice can be built by logically connecting VCCN router instances (see Fig. 1). In a VCCN slice, packet transport between VCCN router instances, which are commonly not adjacent in the $\mathrm{CCN}$ network, is enabled by lower layer protocols than those defining $\mathrm{CCN}$ or by tunneling in the CCN layer [2].

In the following, we describe three types of conceivable methods of allocating CS resources to VCCN router instances: an exclusive method, a shared method and a hybrid method (see Fig. 2).

- Exclusive method

Each VCCN router instance monopolizes a given part of the CS of a CCN router. One advantage of the exclusive method is that the performance of content caching in a VCCN router instance is independent of that in other VCCN router instances. On the other hand, a disadvantage is that the cache miss rate may increase because a VCCN router instance 
monopolizes a given part of the CS regardless of the amount of traffic in the VCCN router instance.

- Shared method

All VCCN router instances jointly use the entire CS of a CCN router. One advantage of the shared method is that there is no loss of CS due to splitting of the CS and the cache hit rate may be increased because all VCCN router instances use the entire CS. On the other hand, a disadvantage is that a VCCN router instance with a large amount of traffic may monopolize most of the CS because each VCCN router instance affects the others within a given CCN router.

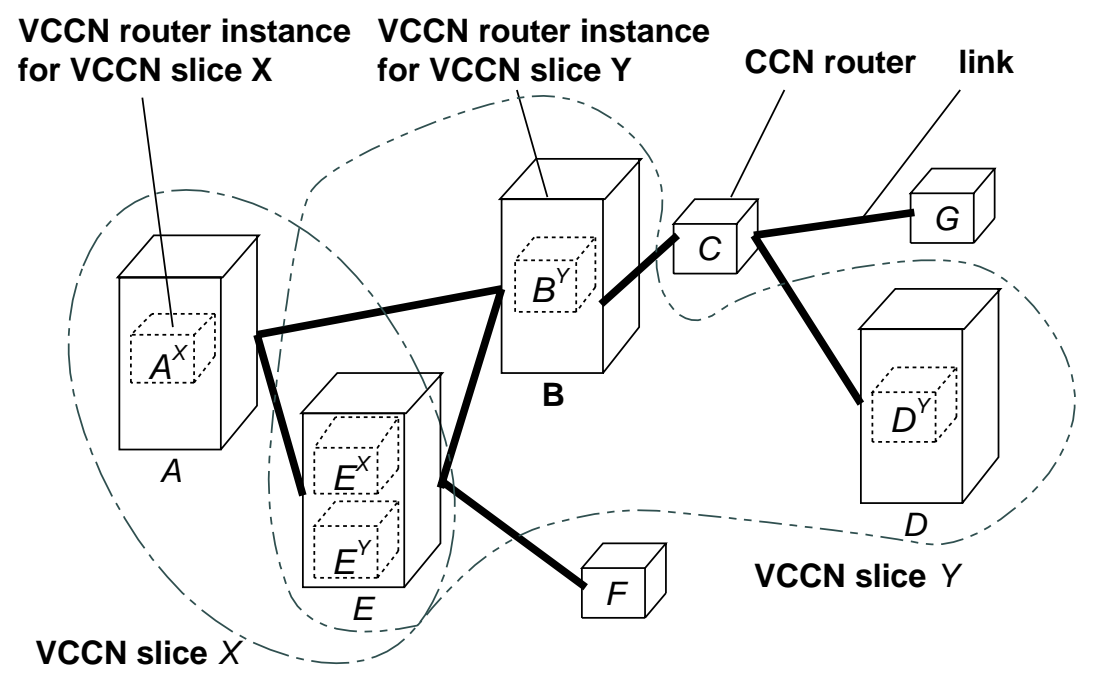

Figure 1: An example of VCCN networks built on a CCN network: two logically independent VCCN networks, X and Y, are built on a network composed of seven CCN routers, A through G.

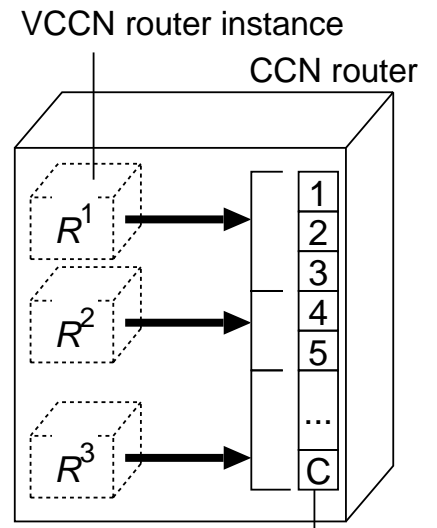

$\mathrm{CS}$ of $\mathrm{CCN}$ router

Exclusive method

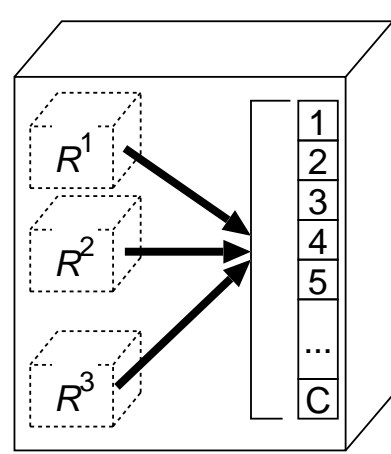

Shared method

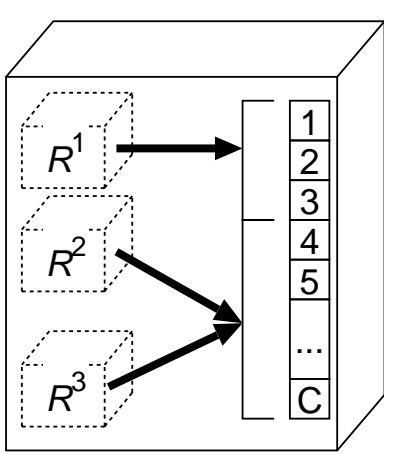

Hybrid method

Figure 2: Examples of methods for the allocation of CS to VCCN routers (an exclusive method, a shared method and a hybrid method).

- Hybrid method 
International Journal of Next-Generation Networks (IJNGN) Vol.5, No.4, December 2013

Several VCCN router instances within a CCN router are assigned their own parts of its CS and other instances jointly use the remaining CS. Advantages of the hybrid method are that CS loss is reduced relative to the exclusive method and each VCCN router instance has a minimal effect on the others within a given $\mathrm{CCN}$ router. On the other hand, a disadvantage is that the management of the CS may be more complicated than in either the exclusive method or the shared method.

Another approach, in which each VCCN router instance monopolizes a small part of the CS and all the instances jointly use the remaining CS, is also conceivable as a hybrid method. However, in this approach, each VCCN router instance needs to be assigned a certain amount of CS. As the number of VCCN router instances running on a CCN router increases, the required size of CS also increases. Hence, this hybrid approach is not considered in this paper.

\section{VIRTUALIZED CCN ROUTER MODEL}

\subsection{Model description and notation}

Our virtualized CCN router model is presented in Fig. 3. VCCN slices $S^{n}(1 \leq n \leq N)$ are constructed on a content-centric network, and VCCN router instances $R^{n}(1 \leq n \leq N)$ corresponding to the VCCN slices operate on a virtualized $\mathrm{CCN}$ router.

We assume that the request arrival process for content $c$ on VCCN router instances $R^{n}$ is Poisson with mean arrival rate $\lambda_{c}^{n}$. Thus, neither the content popularity slope nor the content request ratio for each VCCN slice vary dynamically. $G$ denotes the number of content items requested from all the VCCN router instances.

The CS of the virtualized CCN router is partitioned into $M$ segments and segment $m(1 \leq m \leq M)$ of size $C(m)$ is jointly used by all VCCN router instances belonging to a set $\Omega(m)$. The sets $\Omega(m)$ satisfy the following relations.

$$
\begin{aligned}
\Omega(m) & \subset\left\{R^{1}, R^{2}, \cdots, R^{N}\right\} \\
\bigcup_{m=1}^{M} \Omega(m) & =\left\{R^{1}, R^{2}, \cdots, R^{N}\right\} \\
\sum_{m=1}^{M}|\Omega(m)| & =N
\end{aligned}
$$

So a VCCN router instance does not use multiple segments. Each segment employs a least recently used (LRU) replacement policy.

In our model, each Interest and Data packet has size L in order to simplify the problem. If Data packets have different sizes, we can use the methods of Fricker et al. [11].

Moreover, we do not consider the aggregation of requests for the same content on the virtualized $\mathrm{CCN}$ router because request aggregation has no or little impact on the stationary average content delivery time [12]. When a CCN router receives an Interest packet for content that is already being requested, the $\mathrm{CCN}$ router prevents the dispatch of that Interest packet.

In addition, we assume that the processing times for managing the CS, writing Data packets into the CS and reading Data packets from the CS are negligible. 


\subsection{Determination of the cache hit rate based on a Markov chain model}

First, we derive the cache hit rate $p^{n}$ for each VCCN router instance $R^{n}(1 \leq n \leq N)$ and the aggregated cache hit rate $p$ of the virtualized CCN router based on a Markov chain model. Although it is difficult to derive the performance of a large-scale CCN network in this model because of the huge computational complexity, the state distribution for each successive storage of content in the CS is acquired. Hence, we can estimate the size of CS required to hold a specific amount of content.

Here, we focus VCCN router instances $R^{n} \in \Omega(m)$ which jointly use segment $\mathrm{m}$ of the CS. We denote the state, in which content $c$ on VCCN slice $S^{n}$ is in the kth segment of the CS, by $s_{c, k}$ and we consider the Markov chain composed of $s_{c, k}(0 \leq k \leq C(m))$ (see Fig. 4). We assume without loss of generality that the new content is written into the top of the CS segment. Note that the state in which content $c$ on VCCN slice $S^{n}$ is not in the CS segment is denoted by $s_{c, 0}$ and transitions to the same state are omitted in Fig. 4.

Let $P_{c, k}$ and $P_{c, C(m)}$ be the transition rate from $s_{c, k}$ to $s_{c, k+1}$ and from $s_{c, C(m)}$ to $s_{c, 0}$, respectively.

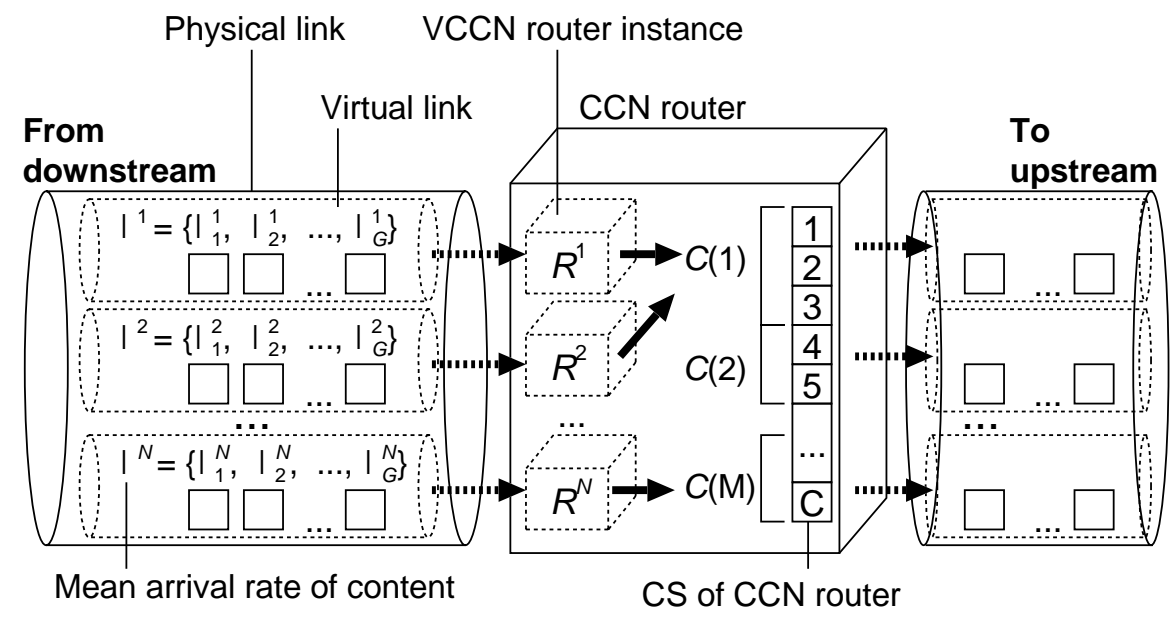

Figure 3: The model considered in this paper.

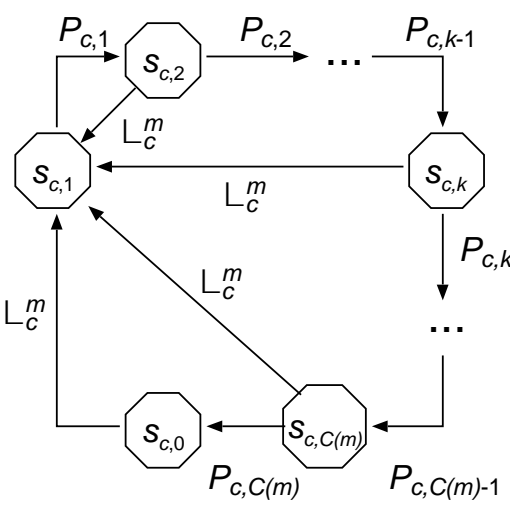

Figure 4: Markov chain model. The state in which content c on VCCN slice $S^{n}$ is placed in the kth segment of the CS is denoted by $s_{c, k}(0 \leq k \leq C(m))$. 
$P_{c, k}$ is given by

$$
P_{c, k}= \begin{cases}\sum_{i=1}^{G} \Lambda_{i}^{m}-\Lambda_{c}^{m} & k=1 \\ \prod_{i=1}^{k} P_{c, i} & 2 \leq k \leq C(m)\end{cases}
$$

where $\Lambda^{m}{ }_{c}$ is the request arrival rate of content $c$ at CS segment $m$ which is given by $\sum_{R^{n} \in \Omega(m)} \lambda_{c}^{n}$

$$
\begin{aligned}
\prod_{i=1}^{k} P_{c, i}= & (k-1) ! \sum_{\Xi \in \Theta_{c}^{k-1}} \prod_{\Lambda_{j}^{i} \in \Xi} \Lambda_{j}^{i} \\
& \times\left(\sum_{i=1}^{G} \Lambda_{i}^{m}-\sum_{\Lambda_{j}^{i} \in \Xi \cup\left\{\Lambda_{c}^{m}\right\}} \Lambda_{j}^{i}\right)
\end{aligned}
$$

where $\Theta^{k}{ }_{c}$ is the set composed of request rates for content in segment $\mathrm{k}$, which satisfies $\Theta^{k} \subset$ $\bigcup_{i=1, i \neq c}^{G} \Lambda_{i}^{m}$ and $\left|\Theta^{k}\right|=k$.

Let $\pi_{c, k}(0 \leq k \leq C(m))$ be the equilibrium probability of $s_{c, k}$. Then $\pi_{c, k}$ is given by

$$
\pi_{c, k}= \begin{cases}\frac{\prod_{i=1}^{C(m)} P_{c, i}}{\prod_{i \bar{\Lambda}_{c}^{m}(m)}^{c}\left(\Lambda_{c}^{m}+P_{c, i}\right)} & k=0 \\ \frac{\Lambda_{c}^{m}}{\Lambda_{c}^{m}+P_{c, 1}} & k=1 \\ \frac{\Lambda_{c}^{m} \prod_{i=1}^{k-1} P_{c, i}}{\prod_{i=1}^{k}\left(\Lambda_{c}^{m}+P_{c, i}\right)} & \text { otherwise }\end{cases}
$$

The cache hit rate $p^{n}{ }_{c}$ of content $c$ on VCCN slice $S^{n}$ can be derived from $\pi_{c, k}$.

$$
p_{c}^{n}=1-\frac{\prod_{i=1}^{C(m)} P_{c, i}}{\prod_{i=1}^{C(m)}\left(\Lambda_{c}^{m}+P_{c, i}\right)}
$$

Finally, the cache hit rate $p^{n}$ for each VCCN router instance $R^{n}(1 \leq n \leq N)$ and the aggregated cache hit rate $p$ of the virtualized CCN router are given by the following equations.

$$
\begin{gathered}
p^{n}=\frac{\sum_{c=1}^{G} \lambda_{c}^{n} p_{c}^{n}}{\sum_{c=1}^{G} \lambda_{c}^{n}} \\
p=\frac{\sum_{n=1}^{N} \sum_{c=1}^{G} \lambda_{c}^{n} p_{c}^{n}}{\sum_{n=1}^{N} \sum_{c=1}^{G} \lambda_{c}^{n}}
\end{gathered}
$$

\subsection{Determination of the hit rate using an approximation method}

We can derive an approximation to the cache hit rate $p^{n}$ for each VCCN router instance $R^{n}(1 \leq n$ $\leq N)$ and the aggregated cache hit rate $p$ of the virtualized CCN router from the hierarchical Web caching model [10]. In this approximation, the network performance of a large-scale CCN network can also be derived. 
If the size of the cache memory is $C$, the cache replacement policy is LRU and the request arrival process for content $c$ is Poisson with mean arrival rate $\lambda_{c}$, the cache hit rate of content $c$ is given by

$$
p_{c} \simeq 1-e^{-\lambda_{c} t_{c}}
$$

where $t_{c}$ is called the characteristic time of content $c$ and is defined as the maximum inter-arrival time between two adjacent requests for content $c$ without a cache miss at the cache [10]. The characteristic time can be calculated by solving

$$
\sum_{i=1, i \neq c}^{G} F_{i}\left(t<t_{c}\right)=C
$$

where $F_{c}\left(t<t_{c}\right)$ is the cumulative distribution $\left(1-\mathrm{e}^{-\lambda c t c}\right)$ of the inter-arrival time for requests for content $c$ at the cache. Without loss of generality, suppose that the caching of content $c$ occurs at $t=0$. Thus, the characteristic time $t_{c}$ is the time at the CS, whose size is $C$, will be filled with content other than $c$. In addition, (10) and (11) can be simplified as follows [11].

where $t_{C}$ is found by solving

$$
p_{c} \simeq 1-e^{-\lambda_{c} t_{C}}
$$

$$
\sum_{j=1}^{G}\left(1-e^{-\lambda_{j} t_{C}}\right)=C
$$

In our model (see Fig. 4.1), the CS of a virtualized CCN router is partitioned into $M$ segments and each segment runs independently. Hence, the cache hit rate for each item of content can be obtained by applying the approximation [10] to each segment.

Thus, the cache hit rate $p^{n}{ }_{c}$ of content $c$ on VCCN slice $S^{n}$ is given by

$$
p_{c}^{n} \simeq 1-e^{-\Lambda_{c}^{m} t_{c}^{m}}
$$

where $t^{m}{ }_{c}$ can be calculated by solving

$$
\sum_{j=1, j \neq c}^{G}\left(1-e^{-\sum_{R^{i} \in \Omega(m)} \lambda_{j}^{i} t_{j}^{m}}\right)=C(m)
$$

These equations can also be simplified to

$$
p_{c}^{n} \simeq 1-e^{-\Lambda_{c}^{m} t^{m}}
$$

where $t^{m}$ is found by solving

$$
\sum_{j=1}^{G}\left(1-e^{-\sum_{R^{i} \in \Omega(m)} \lambda_{j}^{i} t^{m}}\right)=C(m)
$$


From the above, the cache hit rate $p^{n}$ of each VCCN router instance $R^{n}(1 \leq n \leq N)$ and the aggregated cache hit rate $p$ of the virtualized $\mathrm{CCN}$ router are obtained:

$$
\begin{gathered}
p^{n}=\frac{\sum_{c=1}^{G} \lambda_{c}^{n}\left(1-e^{-\Lambda_{c}^{m} t_{c}^{m}}\right)}{\sum_{c=1}^{G} \lambda_{c}^{n}} \\
p=\frac{\sum_{n=1}^{N} \sum_{c=1}^{G} \lambda_{c}^{n}\left(1-e^{-\Lambda_{c}^{m} t_{c}^{m}}\right)}{\sum_{n=1}^{N} \sum_{c=1}^{G} \lambda_{c}^{n}}
\end{gathered}
$$

If (12) and (13) are used, $t_{c}^{m}$ is replaced with $t^{m}$.

\section{NUMERICAL EXAMPLE}

\subsection{Validation of the model}

First, we validated our model by comparing the analytic results of our model with the simulation results. In the determination of the cache hit rate using the approximation method, we used (16) and (17). The network tested is shown in Fig. 5. Three $(N=3)$ VCCN slices $S^{l}, S^{2}$ and $S^{3}$ are constructed on a content-centric network so that we can study three CS allocation methods (i.e., an exclusive method, a shared method and a hybrid method). The link delay between nodes is $10[\mathrm{~ms}]$ irrespective for all slices. In each slice, 10,000 content items $(L=1$ [Mbyte] $)$ are stored in the repository (i.e., $G=30,000$ ). Users generate content requests for each VCCN router instance according to a Poisson process of intensity $\lambda=5[\mathrm{req} / \mathrm{s}]$. The distribution of content popularity is Zipf with parameter $\alpha_{n}$ for VCCN slice $S^{n}$. The content request ratios in all VCCN slices are equal. Here, $\alpha_{1}, \alpha_{2}$ and $\alpha_{3}$ are set for $0.5,0.75$ and 1.5, respectively, by referring to the values of $\alpha$ on existing services [3, 8]. Content requests in VCCN slices do not overlap; this is similar to the situation considered in $[6,8]$. We consider five CS allocation methods: an exclusive method that assigns an equal number of CS segments to each VCCN router instance, a shared method that assigns the entire CS to all VCCN router instances and hybrid methods (Hybrid $\left(S^{n}(1 \leq n \leq 3)\right.$ ) that assign one-third of the CS segments to VCCN router instance $R^{n}$ and assign the remaining segments to the others.

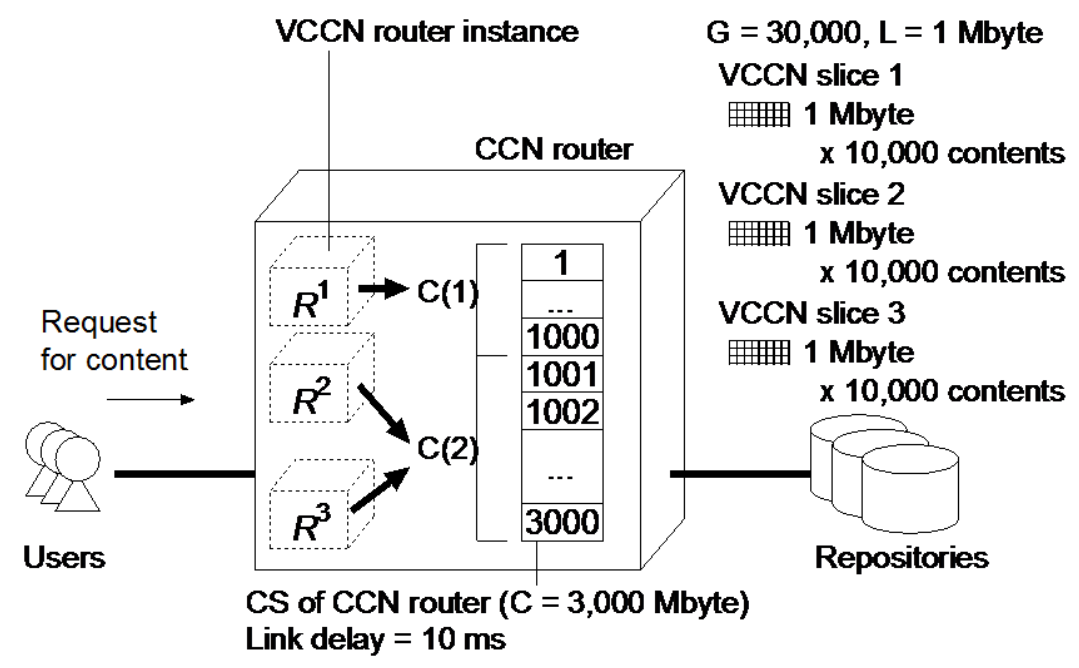

Figure 5: Network used for the evaluation. 
The cache hit rates of VCCN router instance $R^{l}$ and the aggregated cache hit rates of the virtualized CCN router against the size of the CS are shown in Figs. 6 and 7. Fig. 6 shows the results based on the Markov chain model and the simulation results for the exclusive method, the shared method and the hybrid method. In Fig. 6, for convenience of computational complexity, $G$ was set 100 . We confirmed that the tendency of content caching is not very different by this reduction of the number of contents. Fig. 7 shows the results from the approximate analysis and the simulation results for the exclusive method, the shared method and the hybrid method. Since the $95 \%$ confidence intervals of simulation results are too small (all of them are less than $10^{-3}$ ), they are omitted in this paper.

The differences between the analysis results and the simulation results are small in terms of both the VCCN slice's performance and the overall network performance, with a maximum error less than $2 \%$. The results for the other VCCN router instances are similar but are omitted to save space. Moreover, it can be seen from Figs. 6 and 7 that the approximate analysis is as accurate as the Markov chain based analysis.

In all the following results, we convert the cache hit rate into the average content delivery time so that it is easier to understand the direct impact on users. We define the average content delivery time between the users and the virtualized CCN router as $\tau_{l}$ and that between the virtualized CCN router and the repositories as $\tau_{2}$. The average content delivery times $\tau_{1}$ and $\tau_{2}$ include both a transmission delay and a processing delay. The average content delivery time $D$ of the entire network is given by

$$
D=\frac{\sum_{n=1}^{N} \sum_{c=1}^{G} 2 \lambda_{c}^{n}\left(T_{1}+T_{2}\left(1-p_{c}^{n}\right)\right)}{\sum_{n=1}^{N} \sum_{c=1}^{G} \lambda_{c}^{n}}
$$

The analytic solutions for the average content delivery time $D$ of the entire network are also highly accurate, with a maximum error less than $2 \%$.

\subsection{Effects of content popularity slopes in VCCN slices}

Second, we investigated the effects of content popularity slopes in VCCN slices on fairness and overall network performance. Figure 8 shows the average content delivery time of the entire network and the fairness index [13] for VCCN slices against the difference of Zipf exponents $\alpha$ between VCCN slices. Specifically, the figure shows the result of approximate analysis when $\alpha_{1}=$ $1-d, \alpha_{2}=1$ and $\alpha_{3}=1+d$ for $(0 \leq d \leq 1)$.

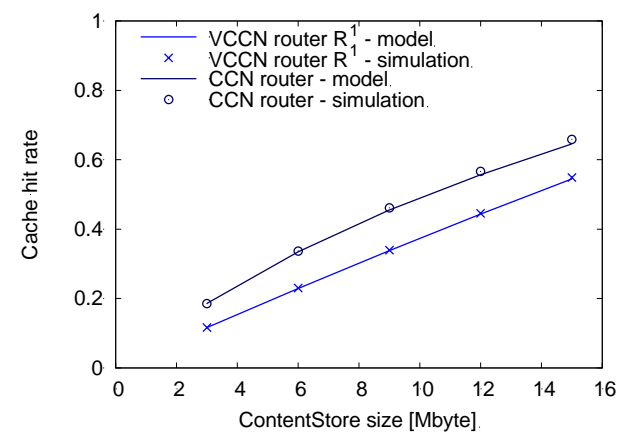

(a) Exclusive

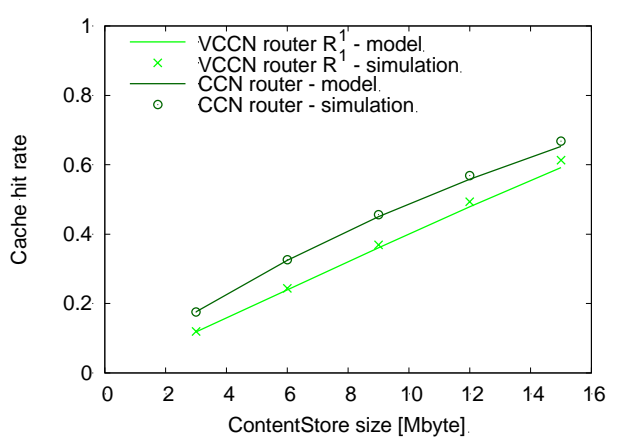

(b) Shared 


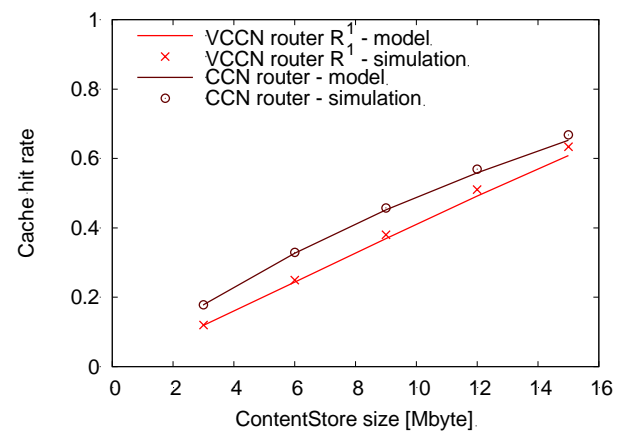

(c) $\operatorname{Hybrid}\left(S^{2}\right)$

Figure 6: The cache hit rate against the size of CS for the exclusive method, the shared method and a hybrid method (Markov chain based analysis).

Figure 8 shows that the overall network performance hardly depends on CS allocation methods and that the shared method is preferable for improving fairness, on a content-centric network in which there are slices with different content popularity slopes. As the difference between the content popularity slopes increases, the size of the CS that $S^{3}$ (with high $\alpha$ ) requires to achieve a high cache hit rate decreases and the size of the CS that $S^{l}$ (with low $\alpha$ ) requires increases. Hence, as the difference between the content popularity slopes increases, an exclusive method cannot keep up with the change of CS size required for each VCCN router instance and the fairness index is degraded. At the least, this result indicates that when the difference of $\alpha$ between any slices is 0.4 or more, the simple exclusive method is not suitable in terms of both fairness and overall network performance.

\subsection{Effects of content popularity slopes in VCCN slices}

Third, we investigated the effects of content request ratios in VCCN slices on fairness and overall network performance. Figure 9 shows the average content delivery time for the entire network and the fairness index against the ratio $r(0 \leq r \leq 1)$ of content requests for VCCN slice $S^{l}$ compared with all requests. Specifically, the figure shows the results of the approximate analysis when the content request ratios of VCCN slices $S^{l}, S^{2}, S^{3}$ are respectively $r,(1-r) / 2$ and $(1-r) / 2$, and $\alpha=0.75$ for all the VCCN slices.

Figure 9 shows that, on a content-centric network in which slices have very different content request ratios, while a shared method is preferable for maximizing the performance of the entire network, an exclusive method is preferable for improving fairness. This result is consistent with existing studies $[6,7,9]$. When the content request ratio of $\operatorname{VCCN}$ slice $S^{l}$ is higher than that of the other slices (i.e., $r \geq 0.4$ ), as $r$ increases, the average content delivery time for the entire

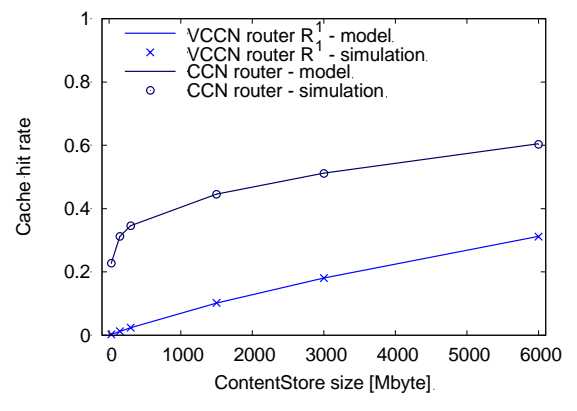

(a) Exclusive

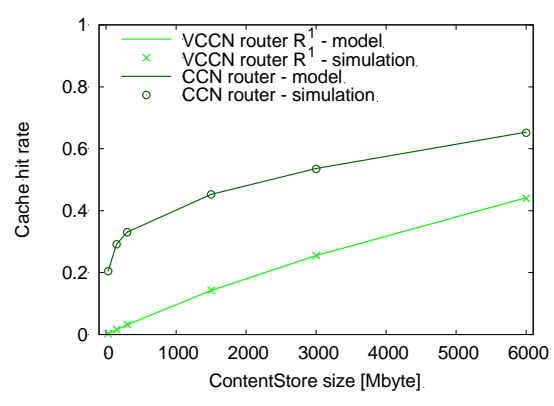

(b) Shared 


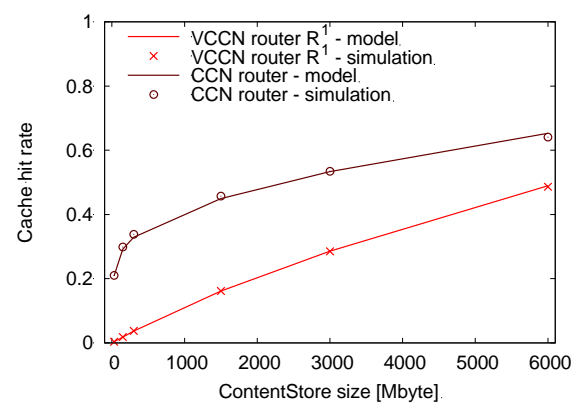

(c) $\operatorname{Hybrid}\left(S^{2}\right)$

Figure 7: The cache hit rate against the size of CS for the exclusive method, the shared method and a hybrid method (approximate analysis).

network decreases for the shared method and the fairness index increases for the exclusive method. On the other hand, Fig. 9 also shows that the hybrid methods $\left(\operatorname{Hybrid}\left(S^{2}\right)\right.$ and Hybrid $\left(S^{3}\right)$ ), which assign a part of the CS to slices other than $S^{1}$, achieve moderate performance in terms of both metrics. At the least, this result indicates that when the content request ratio of a specific slice is higher than that of the other slices, a hybrid method which assigns a part of the CS to a slice with low content request ratio, is preferable for providing a balance between the two metrics. From the above results we conclude that, when content request ratios in VCCN slices are different, the hybrid method is best suited for providing a balance between fairness and overall network performance.

\subsection{Effects of content request patterns in VCCN slices}

Finally, we investigated the combined effects of content popularity slope and content request ratio in each VCCN slice on fairness and overall network performance. Figure 10 shows the average content delivery time for the entire network and the fairness index against content request ratio $r$ $(0 \leq r \leq 1)$ for VCCN slice $S^{l}$. The figure shows the results of the approximate analysis when $\alpha_{l}$, $\alpha_{2}$ and $\alpha_{3}$ are $0.5,0.75$ and 1.5 , respectively.

Figure 10 shows that, on a content-centric network in which there are slices with widely different content popularity slopes and content request ratios the shared method is preferable for maximizing the performance of the entire network, while a hybrid method $\left(\operatorname{Hybrid}\left(S^{2}\right)\right)$ is preferable for improving fairness. As the content request ratio of $S^{l}$ (which has low $\alpha$ ) increases, the size of the CS required by $R^{l}$ is much larger than when only the content request ratio of $S^{l}$ differs. Hence, the fairness index for the shared method is degraded due to the occupancy of the CS by $R^{l}$, and that for the exclusive method is degraded for the reason given in Section 5.2. In

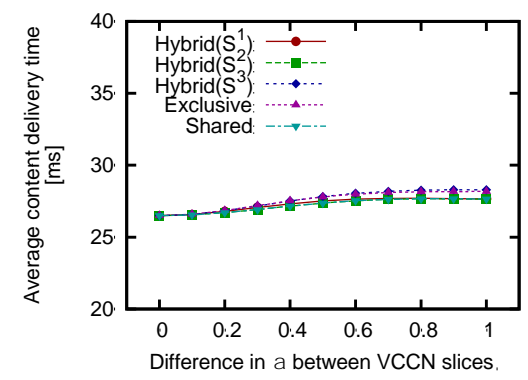

(a) Average content delivery time of the entire network

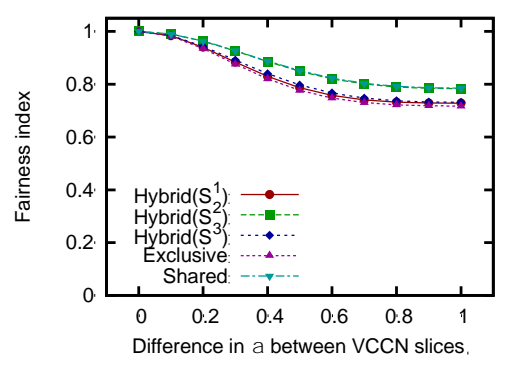

(b) Fairness index for VCCN slices

Figure 8: Average content delivery time and fairness index against the difference of Zipf exponent $\alpha$ between VCCN slices, for each allocation method. 


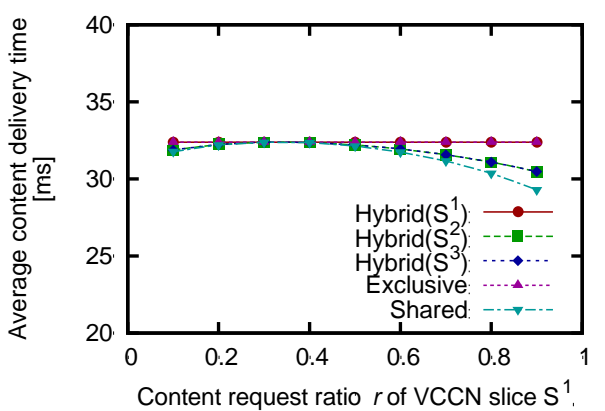

(a) Average content delivery time of the entire network

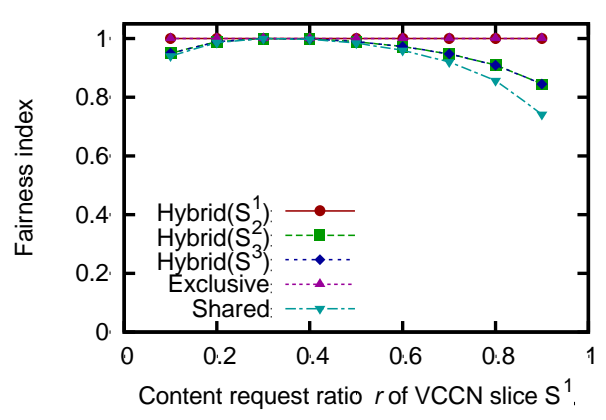

(b) Fairness index for VCCN slices

Figure 9: Average content delivery time and fairness index against the ratio $r$ of content requests for VCCN slice $S^{l}$ compared to all requests for each allocation method.

this case, in order to improve fairness, $R^{l}$ and $R^{3}$, which require a small CS to achieve a high cache hit rate, should jointly use a part of CS, and $R^{2}$ should monopolize the remaining CS. At the least, this result indicates that when the difference of $\alpha$ between any slices is approximately 1.0 and the content request ratio of a slice with low $\alpha$ is higher than those of the other slices (i.e., $r \geq$ 0.4 ), the hybrid method which assigns a part of CS to a slice with a low content popularity slope and low content request ratio, is preferable for improving fairness. From the above results, when both content popularity slopes and content request ratios in VCCN slices are different, the hybrid method is best suited for providing a balance between fairness and overall network performance.

For the hybrid methods, we also investigated the effect of the size of the CS assigned to a VCCN slice on fairness and overall network performance. We used three CS allocation methods: $\operatorname{Hybrid}\left(S^{n}(1 \leq n \leq 3)\right)$ that assign a CS segment whose size is $V$ to VCCN router instance $R^{n}$ and assign the remaining CS to the others. Figure 11 shows the average content delivery time for the entire network and the fairness index against $V$. Specifically, the figure shows the results of the approximate analysis when $r, \alpha_{1}, \alpha_{2}$ and $\alpha_{3}$ are 0.9, 0.5, 0.75 and 1.5, respectively.

Figure 11 shows that several hybrid methods perform well over a wide range of $V$. If $V<2000$ on $\operatorname{Hybrid}\left(S^{2}\right)$ or $1000<V$ on $\operatorname{Hybrid}\left(S^{l}\right)$, the hybrid methods achieve higher performance than the exclusive method on both metrics. In particular, Hybrid $\left(S^{2}\right)$, which assigns a part of the CS to a slice with low content popularity slope and low content request ratio, can most efficiently balance both metrics when the part of the CS allocated to a slice is approximately one third (in general, $1 / N$ ) of the whole CS. From this result, we anticipate that even if the method used to

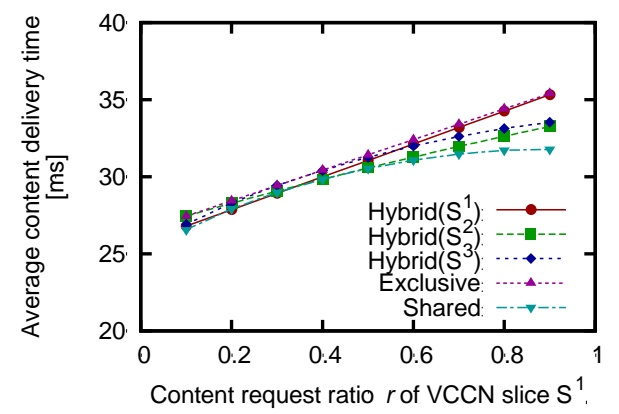

(a) Average content delivery time of the entire network

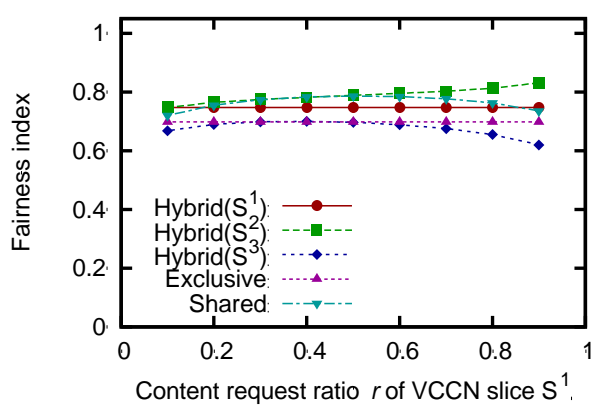

(b) Fairness index for VCCN slices

Figure 10: Average content delivery time and fairness index against content request ratio $\mathrm{r}$ of VCCN slice $S^{l}$ for each allocation method when there is a difference of Zipf exponent $\alpha$ between VCCN slices. 
International Journal of Next-Generation Networks (IJNGN) Vol.5, No.4, December 2013

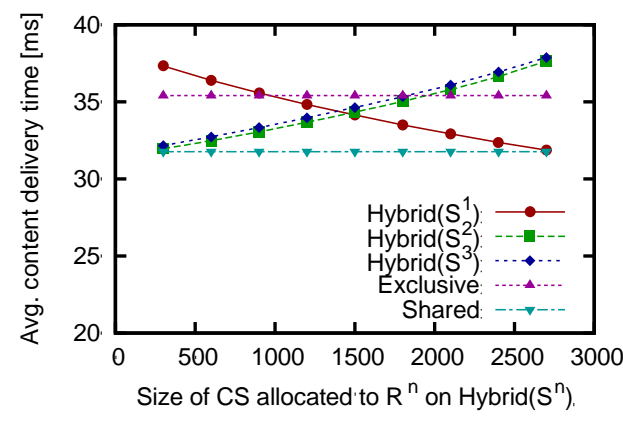

(a) Average content delivery time of the entire network

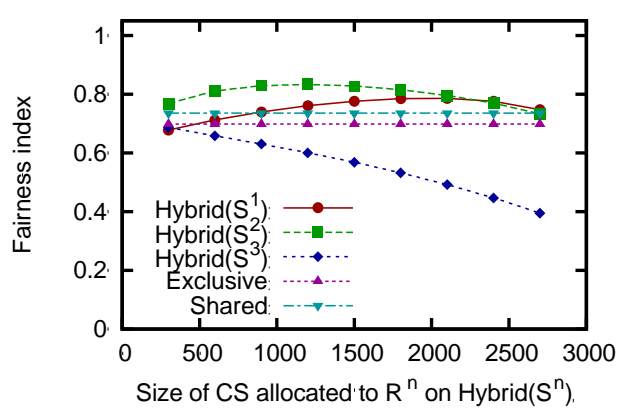

(b) Fairness index for VCCN slices

Figure 11: Average content delivery time and fairness index against the size of CS allocated to $R^{n}$ by $\operatorname{Hybrid}\left(S^{n}\right)$.

allocate the CS to VCCN slices is very simple, a hybrid method will be effective in terms of both fairness and overall network performance.

Generally speaking, content request patterns in VCCN slices (i.e., the content popularity slope and content request ratio for each VCCN slice) will be different. From the above results, the method for allocating CS resources to VCCN routers should be selected as follows (see Table 1). When the difference between content request patterns in VCCN slices is large, a hybrid method that assigns a part of the CS to a slice with low content popularity slope and low content request ratio will provide a balance between the two metrics. On the other hand, whenever fairness between VCCN slices is not important for any content request patterns in the VCCN slices, the shared method will maximize the performance of the entire network.

Table 1: The CS allocation method that should be selected under arbitrary content request patterns in terms of trade-offs among the network fairness for VCCN slices and overall network performance

\begin{tabular}{|l|l|l|l|}
\hline \multicolumn{2}{|c|}{} & \multicolumn{2}{|l|}{$\begin{array}{l}\text { Difference in } \\
\text { content popularity slopes }\end{array}$} \\
\cline { 3 - 4 } \multicolumn{2}{|c|}{} & Negligible & Significant \\
\hline \multirow{2}{*}{$\begin{array}{l}\text { Difference in } \\
\text { content request ratios }\end{array}$} & Negligible & Shared & Shared \\
\cline { 2 - 4 } & Significant & Hybrid & Hybrid \\
\hline
\end{tabular}

\section{Conclusions}

In this paper, we have analytically and quantitatively investigated the effects of CS allocation methods and content request patterns in VCCN slices on network fairness for VCCN slices and the overall network performance. We developed a mathematical model of virtualized $\mathrm{CCN}$ router for cache performance analysis under arbitrary content request patterns, and derived the cache hit rate for each VCCN router instance and the aggregated cache hit rate of the virtualized CCN router. Furthermore, using several numerical examples, we have shown that when content request patterns are heterogeneous, a hybrid resource allocation method will provide the best balance between fairness and overall network performance. 
International Journal of Next-Generation Networks (IJNGN) Vol.5, No.4, December 2013

In the future we will extend our model to investigate the effect of network topology in a network composed of multiple virtualized CCN routers. We will also develop a dynamic CS allocation method, which operates efficiently in a distributed environment in which both the content popularity slope and the content request ratio of each VCCN slice are dynamically varying.

\section{ACKNOWLEDGEMENTS}

This research was partly supported by a Grant-in-Aid for Scientific Research (A) (24240010) and (B) (25280030).

\section{REFERENCE}

[1] V. Jacobson, D. K. Smetters, J. D. Thornton, M. F. Plass, N. H. Briggs, and R. L. Braynard, "Networking named content," in Proc. the fifth International Conference on emerging Networking EXperiments and Technologies (CoNEXT '09), Dec. 2009, pp. 1-12.

[2] M. Ohtani, K. Tsukamoto, Y. Koizumi, H. Ohsaki, K. Hato, J. Murayama, and M. Imase, "VCCN: virtual content-centric networking for realizing group-based communication," in Proc. 12th IEEE International Conference on Communications (ICC '13), June 2013, pp. 2069-2073.

[3] G. Rossini and D. Rossi, "A dive into the caching performance of content centric networking," http://perso.telecom-paristech.fr/ drossi/paper/rossi11ccn-techrep2.pdf, Telecom ParisTech, Tech. Rep., 2011.

[4] D. Rossi and G. Rossini, "Caching performance of content centric networks under multi-path routing (and more)," http://perso.telecom-paristech.fr/ drossi/paper/rossi11ccn-techrep1.pdf, Telecom ParisTech, Tech. Rep., 2011.

[5] I. Psaras, R. G. Clegg, R. Landa, W. K. Chai, and G. Pavlou, "Modelling and evaluation of CCNcaching trees," in Proc. the 10th international IFIP TC 6 conference on Networking - Volume Part I (Networking '11), May 2011, pp. 78-91.

[6] G. Carofiglio, V. Gehlen, and D. Perino, "Experimental evaluation of memory management in content- centric networking," in Proc. 10th IEEE International Conference on Communications (ICC '11), June

2011, pp. 1-6.

[7] G. Carofiglio, M. Gallo, L. Muscariello, and D. Perino, "Evaluating per-application storage management in content-centric networks," Elsevier Computer Communications, vol. 36, no. 7, pp. 750-757, Apr. 2013.

[8] C. Fricker, P. Robert, J. Roberts, and N. Sbihi, "Impact of traffic mix on caching performance in a content-centric network," in Proc. First Workshop on Emerging Design Choices in Name-Oriented Networking at IEEE INFOCOM 2012 (NOMEN'12), Mar. 2012, pp. 310-315.

[9] K. Ohsugi, K. Tsukamoto, and H. Ohsaki, "Study on the effect of CCN router virtualization on content delivery time (in japanese)," IEICE Society Conference 2012, B-7-4, Aug. 2012.

[10] H. Che, Y. Tung, and Z. Wang, "Hierarchical Web caching systems: Modeling, design and experimental results," IEEE J. Selected Areas in Communications, vol. 20, no. 7, pp. 1305-1314, Sept. 2002.

[11] C. Fricker, P. Robert, and J. Roberts, "A versatile and accurate approximation for LRU cache performance," in Proc. 24th International Teletraffic Congress (ITC '12), Sept. 2012, pp. 1-8.

[12] G. Carofiglio, M. Gallo, L. Muscariello, and D. Perino, "Modeling data transfer in content-centric networking," in Proc. the 23rd International Teletraffic Congress (ITC '11), Sept. 2011, pp. 111-118.

[13] R. K. Jain, D.-M. W. Chiu, and W. R. Hawe, "A quantitative measure of fairness and discrimination for resource allocation in shared computer systems," http://www1.cse.wustl.edu/ jain/papers/ftp/fairness.pdf, Digital Equipment Corporation, Tech. Rep. DEC-TR-301, 1984. 


\section{Authors}

Keiichiro Tsukamoto received Bachelor of Information Science and Master of Information Science degrees from Osaka University in 2009 and 2011, respectively. He has been a graduate student of the Graduate School of Information Science and Technology, Osaka University since April 2011. His research interests are in the area of Web content mining and Content-Centric Networking. He is a member of IEICE.

Kaito Ohsugi received Bachelor of Information Science from Osaka University in 2013. He has been a graduate student of the Graduate School of Information Science and Technology, Osaka University since April 2013. He is a member of IEICE.

Hiroyuki Ohsaki received the M. E. degree in the Information and Computer Sciences from Osaka University, Osaka, Japan, in 1995. He also received the Ph. D. degree from Osaka University, Osaka, Japan, in 1997. He is currently a professor at Department of Informatics, School of Science and Technology, Kwansei Gakuin University, Japan. His research work is in the area of design, modeling, and control of large-scale communication networks. He is a member of IEEE, IEICE, and IPSJ.

Toru Hasegawa received the B.E., the M.E. and Dr. Informatics degrees in information engineering from Kyoto University, Japan, in 1982, 1984 and 2000, respectively. Since joining KDD (currently KDDI) in 1984, he has been working in the field of formal description technique (FDT) of communication protocols, ATM and high-speed protocols. From 1990 to 1991, he was a visiting researcher at Columbia University. His current interests are future Internets, Information Centric Networking, mobile computing and so on. He is currently a professor an Osaka university. He received the Meritorious Award on Radio of ARIB in 2003. He is a fellow of IPSJ and IEICE.

Masayuki Murata received the M.E. and D.E. degrees in Information and Computer Science from Osaka University, Japan, in 1984 and 1988, respectively. In April 1984, he joined Tokyo Research Laboratory, IBM Japan, as a Researcher. From September 1987 to January 1989, he was an Assistant Professor with Computation Center, Osaka University. In February 1989, he moved to the Department of Information and Computer Sciences, Faculty of Engineering Science, Osaka University. In April 1999, he became a Professor of Cybermedia Center, Osaka University, and is now with Graduate School of Information Science and Technology, Osaka University since April 2004. His research interests include computer communication networks, performance modeling and evaluation. He is a member of IEEE, ACM and IEICE.
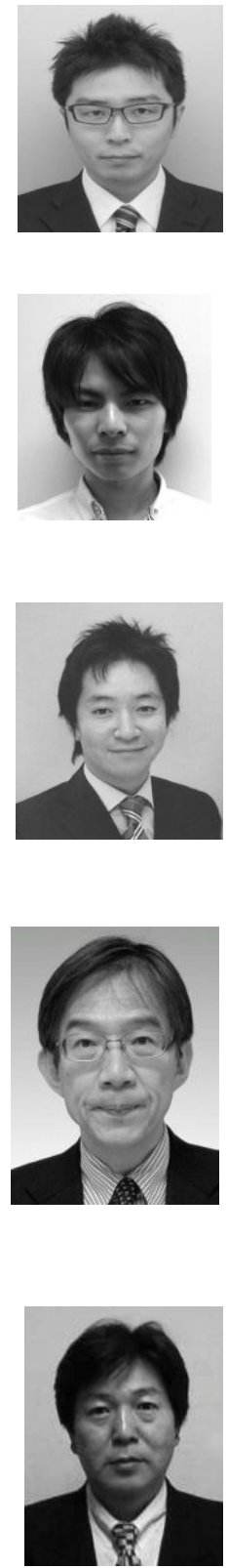